\title{
La réaction au dégagement et les caractéristiques individuelles du noyer cendré favorisant la résistance au chancre causé par l'Ophiognomonia clavigignenti-juglandacearum
}

\author{
par Pierre DesRochers ${ }^{1, *}$, Nicolas Nadeau-Thibodeau ${ }^{2,3}$ Louis Bernier $^{2}$ et Danny Rioux ${ }^{1}$
}

\begin{abstract}
RÉSUMÉ
Nous examinons l'impact du dégagement et des caractéristiques des noyers cendrés, y compris le phénotype d'écorce, sur leur santé. Sur deux sites au Québec, des noyers ont été mis en lumière par détourage de cime. Nous analysons aussi les caractéristiques de noyers recueillies par un collaborateur en Montérégie en lien avec leur santé. Il n'a pas été démontré que ce dégagement améliorait la santé des noyers. Cependant, le site, la croissance en diamètre avant le dégagement et le prélèvement de rameaux sur certains noyers ont eu un impact significatif sur certaines variables de santé. Concernant les noyers cendrés de la Montérégie, les dommages au tronc et la résistance potentielle au chancre causé par l'Ophiognomonia clavigignenti-juglandacearum sont influencés de façon significative par l'étage auquel ils appartiennent, une position supérieure garantissant une meilleure santé. La mort en cime des noyers de phénotype à écorce foncée et rugueuse est significativement moindre que celle des noyers à écorce lisse. La vigueur des noyers est supérieure chez les arbres présentant une plus grande croissance annuelle en surface terrière. Cette croissance annuelle est significativement plus élevée chez les noyers des étages supérieurs et chez les noyers cendrés à écorce rugueuse.
\end{abstract}

Mots clés : noyer cendré, Ophiognomonia clavigignenti-juglandacearum, dégagement, chancre, résistance, mort en cime, vigueur, croissance radiale, phénotype d'écorce, étage, surface terrière

\begin{abstract}
This research examines the impact of light and tree attributes of butternuts, including the bark phenotype, on their health. Some butternuts were released by thinning the crown of neighbour trees in two locations in Québec, while others were not. Various butternut characteristics related to their health that had been collected by a partner in the Montérégie region are also analysed. The release treatment did not have any significant impact on any health variables. However, the location, the diameter increments before releasing trees and twig collection on some butternuts had a significant impact on some of these variables. For the butternuts of the Montérégie region, the story they belong to had a significant impact on the main stem damage and on the putative resistance to the canker caused by Ophiognomonia clavigignenti-juglandacearum, the trees from the highest story showing better health. Dieback of dark and deep furrowed bark phenotype butternuts was significantly lower than that of the light shallow bark ones. Butternuts with a greater annual increase in basal area had better vigour. This annual increase was greater in the higher stories and among the deep furrowed bark butternuts.
\end{abstract}

Keywords: butternut, Ophiognomonia clavigignenti-juglandacearum, release, canker, resistance, dieback, vigour, growth increment, bark phenotype, story, basal area

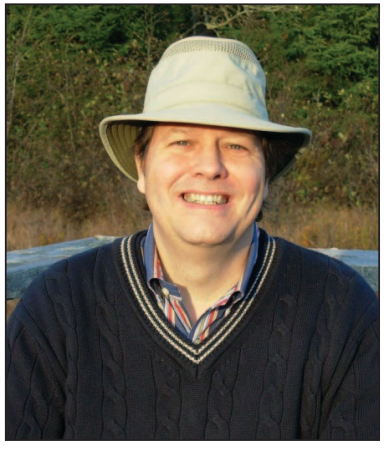

Pierre DesRochers

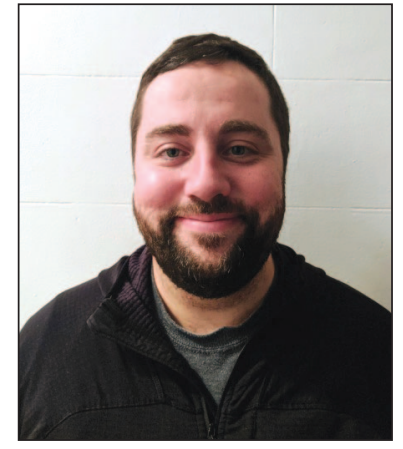

Nicolas Nadeau-Thibodeau

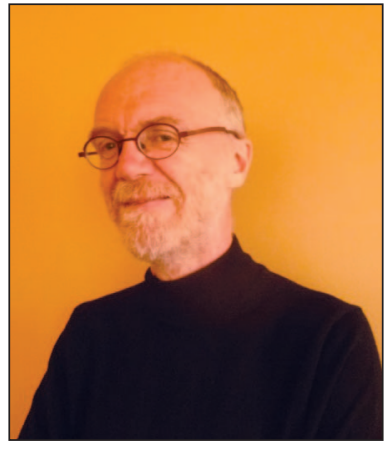

Louis Bernier

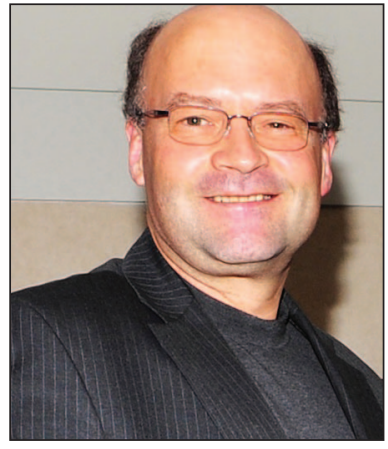

Danny Rioux

\footnotetext{
${ }^{1}$ Centre de foresterie des Laurentides, Service canadien des forêts, Ressources naturelles Canada, 1055, rue du P.E.P.S., C.P. 10380, succ. Sainte-Foy, Québec, QC G1V 4C7; * Auteur de correspondance : pierremdesrochers@gmail.com

${ }^{2}$ Centre d'étude de la forêt, Faculté de foresterie, de géographie et de géomatique, Université Laval, Québec, QC G1V 0A6, Canada

${ }^{3}$ Adresse actuelle : Ville de Montréal, Arrondissement Le Plateau-Mont-Royal, 201 Avenue Laurier Est, H2T 3E6
} 


\section{Introduction}

Le noyer cendré est une composante mineure de la forêt feuillue des vallées du Saint-Laurent, de l'Outaouais et des Grands-Lacs (Grandtner 1966). Au Québec, la station la plus nordique connue se situe au Lac Tapani, au nord-est du Réservoir Baskatong (Majcen 1995). On le trouve aussi au Nouveau-Brunswick (Farrar 1996). Il est présent également dans une trentaine d'États américains (Rink 1990). Au Québec, il croît généralement au sein d'associations forestières telles l'érablière à caryer, l'érablière à tilleul et l'érablière à bouleau jaune (Lupien 2013), où on le retrouve isolé ou en petits groupes, couvrant $3 \%$ ou moins du couvert forestier (Grandtner 1966). Il forme occasionnellement de petits peuplements dans lesquels il occupe jusqu'à la moitié de la surface terrière, mais alors généralement que dans les strates arborées haute et moyenne (Majcen 1995). Cependant, même lorsque les peuplements comprennent une forte proportion de noyers cendrés, ceux-ci sont généralement absents de la strate arborée basse et des strates arbustives (Majcen 1995) ou de la régénération (Grandtner 1966). En effet, l’absence de lumière nuit à la reproduction de cette essence de moyenne grandeur et de faible longévité (75 ans) intolérante à l'ombre (Rink 1990; Niinemets et Valladares 2006).

Outre l'absence de lumière dans les strates inférieures, plusieurs ravageurs menacent la santé et la survie du noyer cendré. Déjà, Graves (1923) identifiait le chancre mélanconien causé par le Melanconis juglandis (Ellis \& Everh.) A.H. Graves comme causant un lent dépérissement du noyer cendré. Les noyers les plus sensibles à cet agent pathogène sont ceux dont la vigueur est moindre, en particulier ceux qui manquent de lumière (Graves 1923). D'autres ravageurs peuvent affecter la santé et la survie de cette essence : l'anthracnose causée par le Marssonina juglandis (Lib.) Magnus et les défoliateurs comme la chenille à tente estivale (Hyphantria cunea Drury), la pyrale (Acrobasis demotella Grote) et la chenille du noyer (Datana integerrima Grote \& Robinson) qui réduisent la capacité photosynthétique des noyers, leur vigueur et leur capacité à réagir aux stress (Farris et Appleby 1979; Nielsen et al. 2003; Environnement Canada 2010). D'autres ravageurs s'attaquent spécifiquement aux tissus ligneux, notamment le pourridié causé par l'Armillaria gallica H. Marxm. \& Romagn., le charançon du noyer (Conotrachelus juglandis Lec.) et les chancres phomopsien et fusarien (Corneil et Wilson 1979; Rink 1990; Nielsen et al. 2003; Environnement Canada 2010), provoquant la mortalité de rameaux et de branches.

Cependant, le chancre du noyer cendré causé par l'Ophiognomonia clavigignenti-juglandacearum (Nair, Kostichka \& Kuntz) Broders \& Boland demeure la plus grande menace contre cette essence (Nair et al.1979; Environnement Canada 2010; Broders et Boland 2011). Observé pour la première fois au Wisconsin en Amérique du Nord en 1967 (Ostry et Woeste 2004), il a été détecté au Québec en 1990 (Innes et Rainville 1996), en Ontario en 1991 (Davis et al. 1992), et au Nouveau-Brunswick en 1997 (Harrison et al. 1998). Bien que l'origine du champignon soit inconnue, sa faible variabilité génétique et sa virulence portent à croire qu'il s'agit d'un ravageur exotique (Furnier et al. 1999). Des travaux récents montrent par ailleurs que la population d'O. clavigignentijuglandacearum se compose de trois groupes d'isolats différents correspondant à trois introductions distinctes, ou des isolats qui ont évolués à partir de formes avirulentes chez le noyer cendré (Broders et al. 2012).

Le champignon pénètre d'abord dans des rameaux, par les bourgeons, les cicatrices foliaires, les lenticelles ou les blessures dans l'écorce (Kuntz et al. 1979; Ostry 1997; Sinclair et Lyon 2005). Ces rameaux dépérissent et se couvrent de colonnes stromatiques qui produisent des conidies capables d'infecter les branches et les troncs de leur hôte et les noyers voisins. Les noyers infectés présentent une quantité croissante de rameaux dépérissants ou morts dans leur cime. Au fur et à mesure que l'infection au tronc progresse, les arbres s'affaiblissent et meurent. Certains auteurs ont suggéré l'existence d'un phénotype de noyers cendrés peut-être résistants à l'O. clavigignenti-juglandacearum (Ostry et al. 2003; Michler et al. 2006). Ces noyers ont une écorce plus foncée présentant des crevasses plus profondes. Selon Boraks et Broders (2014), même si les noyers cendrés à écorce lisse présentaient plus de dommages au tronc, que ceux à écorce rugueuse et profondément crevassée, cette différence pourrait être le résultat du diamètre plus petit des noyers cendrés à écorce lisse. Enfin, la mortalité croissante des noyers cendrés au Canada a fait en sorte qu'il soit déclaré espèce en voie de disparition en 2005 et un programme de rétablissement a été proposé quelques années plus tard (Environnement Canada 2010).

Ce programme prévoit notamment que : « D'ici 2019, aborder les principales lacunes dans les connaissances et les recherches nécessaires à la mise en œuvre des activités de rétablissement (notamment les recherches sur la résistance à la maladie, sur le niveau de variation génétique adaptative et sur les facteurs environnementaux limitant la propagation de la maladie). " La maladie dont il est question est le chancre causé par l'O. clavigignenti-juglandacearum que nous désignerons seulement par le chancre dans la suite de cet article.

Il existe des recommandations sylvicoles concernant le noyer cendré (Lupien 2006), mais celles-ci visent la production de bois de qualité et l'établissement de la régénération. Ostry et al. (1994) ont émis des recommandations quant à la conservation des noyers cendrés potentiellement résistants : conserver les noyers sans dommage au tronc présentant moins de $50 \%$ de mort en cime et aussi ceux présentant moins de $30 \%$ de mort en cime mais avec moins de $20 \%$ de la circonférence du tronc touchée par le chancre. McIlwrick et al. (2000) recommandent d'éliminer les noyers morts le plus rapidement possible, compte tenu de leur capacité à transmettre $O$. clavigignenti-juglandacearum aux noyers sains dans leur voisinage. Des pratiques sylvicoles ont été suggérées pour réduire l'impact de différents chancres, soit par éclaircie ou par élagage. Ainsi, Amorini et al. (2001) ont démontré que l'éclaircie autour de châtaigniers sains permettait de réduire la prévalence du chancre causé par le Cryphonectria parasitica (Murrill) M. E. Barr. Une observation similaire a été rapportée concernant la mortalité causée par diverses maladies du peuplier faux-tremble, à l'exception du chancre hypoxylonien (Pitt et al. 2001). Il existe aussi plusieurs publications sur les effets bénéfiques de l'élagage des rameaux chancrés sur la santé et la survie des arbres atteints (Abgrall et Soutrenon 1991; Laflamme 1999; Jacobi et al. 2017; Weber 2014). Nous n'avons pas trouvé de tels travaux concernant le noyer cendré. Compte tenu de l'intolérance à l'ombre du noyer cendré (Niinemets et Valladares 2006), l'ac- 
cès à la lumière pourrait être un facteur influençant la résistance au chancre. D’ailleurs, les données préliminaires récoltées lors d'un inventaire effectué de 2006 à 2008 (DesRochers et al. 2010) tendaient à démontrer que les arbres poussant à découvert ou dans les étages supérieurs du couvert forestier étaient en meilleure santé que les arbres se développant à l'ombre. Dans ce contexte, les objectifs de cette recherche sont :

1. Examiner l'impact de la mise en lumière sur les variables de santé des noyers cendrés et la résistance au chancre;

2. Et alternativement, examiner l'influence d'autres caractéristiques individuelles des noyers cendrés sur leur santé, notamment le phénotype d'écorce.

\section{Matériel et méthodes Les études}

Nous rapportons ici les résultats de deux études. Tout d'abord une expérience de mise en lumière des noyers cendrés a été réalisée en 2011 de façon à augmenter la lumière dont bénéficient les noyers. Dans un deuxième temps, nous analysons les données recueillies par un collaborateur en Montérégie, CIME Haut-Richelieu, entre 2005 et 2016.

L'expérience de mise en lumière a été réalisée sur deux sites au Québec, à la station agronomique de l'Université Laval à Saint-Augustin-de-Desmaures et sur le territoire de la Défense nationale au mont Saint-Bruno. Les noyers cendrés sélectionnés appartenaient tous à l'étage dominant ou codominant, à l'exception d'un noyer classé comme intermédiaire. Sur chacun des sites, 20 noyers cendrés potentiellement résistants (Ostry et al. 1994) à l'O. clavigignentijuglandacearum ont été sélectionnés. En 2010, la mort en cime des noyers cendrés sélectionnés variait de nulle à $10 \%$, à l'exception d'un noyer montrant $20 \%$ de mort en cime. Les dommages au tronc étaient nuls ou ne dépassaient pas le quart de la circonférence. Les noyers cendrés du mont SaintBruno mesuraient en moyenne $39,9 \mathrm{~cm}$ de diamètre à hauteur de poitrine (DHP), avec un écart-type de $9,9 \mathrm{~cm}$ (intervalle de $17,5 \mathrm{~cm}$ à $57,8 \mathrm{~cm}$ ), alors que ceux de la Station agronomique mesuraient $39,6 \mathrm{~cm}$ pour un écart-type de 7,7 $\mathrm{cm}$ (intervalle de $27,8 \mathrm{~cm}$ à $61,2 \mathrm{~cm}$ ). Les données regroupées pour ces deux sites, par classes de dominance, sont présentées au Tableau 1. Pour chaque site, deux traitements ont été répartis au hasard entre les arbres, 10 noyers cendrés servant de témoins et les 10 autres traités par une mise en lumière s'apparentant au détourage (Lupien 2006). Aucun dégagement n'a été fait sur les arbres témoins. Pour les noyers mis en lumière, la cime des arbres voisins a été émondée de manière à dégager un espace libre de branches de $5 \mathrm{~m}$ autour des individus sélectionnés Il s'agissait d'une ouverture du couvert, visuellement acceptable pour les nombreux visiteurs et militaires qui fréquentent le mont Saint-Bruno tout au long de l'année. Ces travaux ont été réalisés entre le 20 janvier et le 31 mars 2011. Un noyer du mont Saint-Bruno et un noyer de la Station agronomique ont été exclus des évaluations et de l'analyse parce qu'ils ont été déracinés ou gravement endommagés par la tempête qui a suivi l'ouragan Irène en 2011, l'été après l'exécution des traitements. Il s'agissait de deux noyers témoins. La santé des noyers a été suivie annuellement jusqu'en 2016. Nous analysons les données de santé de 2016 pour les deux traitements réalisés. Après l'installation, notre dispositif a aussi été utilisé par Tanguay et al. (2018) pour leur étude sur la production de conidies d'O. clavigignenti-juglandacearum.

Nous avons fait évaluer quelque 65 noyers cendrés en milieu naturel au Québec (résultats non publiés) et aucun ne présentait de traces d'hybridation avec le noyer japonais $(J$. ailantifolia Carr.). Les hybrides entre ces deux espèces sont réputés plus résistants au chancre que le noyer cendré (Orchard et al. 1982) et il était donc souhaitable de vérifier la pureté de quelques 200 noyers cendrés que nous avions identifiés comme étant potentiellement résistants sur différents sites au Québec (Nadeau-Thibodeau 2015). Plus spécifiquement, et bien que tous les arbres n'aient pas été évalués, une dizaine d'arbres ont été analysés sur chacun des deux sites du mont Saint-Bruno et de Saint-Augustin, dont six simultanément en utilisant quatre marqueurs-microsatellites (J. A. McLaughlin et G. Halicki Hayden, Ministère des Richesses naturelles et des Forêts de l'Ontario, résultats non publiés) et par génotypage à l'aide de séquençage $(\mathrm{N}$. Isabel et $\mathrm{M}$. Lamothe, CFL, résultats non publiés), sans révéler aucune trace d'hybridation. De surcroit, nous avons vérifié les caractères morphologiques des rameaux prélevés sur les 19 noyers de l'expérience de bouturage conduite parallèlement, décrite au paragraphe suivant. Tous ces noyers ne possédaient que les caractères propres au noyer cendré (Woeste et al. 2009).

Une autre expérience a été conduite de façon concurrente sur plusieurs des noyers de cette expérience, compte tenu du nombre limité de noyers cendrés et de façon à utiliser le personnel technique disponible de façon optimale : 30 à 45 rameaux dormants sains par individu ont été récoltés sur 13 noyers témoins et six noyers en détourage de cime, choisis au hasard et répartis également entre les deux sites, pour une expérience de bouturage (Nadeau-Thibodeau 2015, Rioux et al. 2019). De plus, les arboriculteurs ont retiré du bois mort afin de faciliter l'accès aux rameaux sains au sommet de la cime.

Tableau 1. Diamètre à hauteur de poitrine des noyers cendrés de l'expérience de dégagement en fonction de la dominance, mesuré avant le traitement, en 2010

\begin{tabular}{lccccc}
\hline & & \multicolumn{4}{c}{ DHPi (cm) } \\
\cline { 3 - 5 } Étage $^{\text {a }}$ & Nombre & Minimum & Maximum & Moyenne & Écart-type \\
\hline Dominants et à découvert & 19 & 26,7 & 61,2 & 43,8 & 8,6 \\
Codominants et intermédiaires & $18^{\mathrm{b}}$ & 17,5 & 47,0 & 35,7 & 6,9 \\
\hline
\end{tabular}

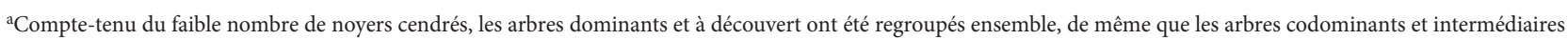

bune donnée manquante 
Tableau 2. Diamètre à hauteur de poitrine initial (DHPi) des noyers cendrés évalués par CIME Haut-Richelieu, en fonction du nombre de saisons de croissance et du phénotype d'écorce

\begin{tabular}{|c|c|c|c|c|c|}
\hline \multirow[b]{2}{*}{ Caractéristique } & \multirow[b]{2}{*}{ Nombre } & \multicolumn{4}{|c|}{ DHPi (cm) } \\
\hline & & Minimum & Maximum & Moyenne & Écart-type \\
\hline \multicolumn{6}{|l|}{ Saisons } \\
\hline Deux & 3 & 6,8 & 15,0 & 10,9 & 4,1 \\
\hline Trois & 4 & 10,8 & 22,0 & 15,4 & 4,8 \\
\hline Quatre & 60 & 0,5 & 95,2 & 19,9 & 16,7 \\
\hline Cinq & 13 & 2,9 & 44,3 & 24,5 & 12,3 \\
\hline Six & 1 & - & - & $20,7-$ & \\
\hline Sept & 4 & 14,6 & 54,5 & 37,6 & 16,8 \\
\hline \multicolumn{6}{|l|}{ Phénotype d'écorce } \\
\hline Lisse & $24 \mathrm{i}$ & 2,9 & 44,5 & 19,3 & 11,1 \\
\hline Intermédiaire & 38 & 0,5 & 47,1 & 16,8 & 11,8 \\
\hline Rugueuse & 23 & 5,0 & 95,2 & 29,5 & 22,1 \\
\hline
\end{tabular}

Par ailleurs, des données sur la croissance et la santé du noyer cendré ont été récoltées sur 85 arbres pendant plusieurs années successives entre 2005 et 2016 au mont SaintGrégoire et sur des terres privées à Saint-Jean-sur-Richelieu, en Montérégie, par les collaborateurs de l'organisme CIME Haut-Richelieu. Le diamètre à hauteur de poitrine (DHP) de ces arbres a été mesuré lors de l'évaluation initiale (Tableau 2) et une seconde fois, quelques années plus tard. L'intervalle entre les deux mesures variait de deux à sept saisons de croissance, selon les sites suivis. Nous avons analysé les données de santé de la dernière année où le DHP a été mesuré. Les noyers suivis par CIME Haut-Richelieu mesuraient en moyenne $20,9 \mathrm{~cm}$ de DHP, à leur première mesure, avec un écart-type de $15,8 \mathrm{~cm}$. Les DHP des noyers de phénotype d'écorce lisse et pâle, intermédiaire ou rugueuse et foncée étaient respectivement de $19,9 \mathrm{~cm} .16,8 \mathrm{~cm}$ et $29,5 \mathrm{~cm}$, avec des écart-types respectifs de $11,1 \mathrm{~cm}, 11,8 \mathrm{~cm}$ et $22,1 \mathrm{~cm}$.

\section{Les variables dendrométriques}

Nous avons évalué les variables suivantes : le diamètre à hauteur de poitrine (DHP) et la dominance. Le DHP est mesuré en centimètres et dixièmes de centimètres, au gallon circonférentiel, à une hauteur de 1,3 m au-dessus du sol selon la méthode du Dispositif national d'alerte rapide pour les pluies acides (DNARPA; D'Eon et al. 1995).

La dominance, a été mesurée selon la méthodologie du DNARPA (D’Eon et al. 1995). Les arbres sont classés comme dominants (valeur 1) s'ils sont à découvert ou situés dans la partie supérieure du couvert forestier et reçoivent de la lumière par le haut et les côtés; les arbres codominants (valeur 2) reçoivent de la lumière principalement par le haut; les arbres intermédiaires (valeur 3 ) sont plus petits que les arbres codominants et reçoivent peu de lumière alors que les arbres opprimés (valeur 4) sont entièrement situés sous le couvert forestier.

La croissance radiale périodique, en $\mathrm{mm}$, avant le traitement de 2011, a été calculée pour la période de 2005 à 2010 pour tous les noyers de l'expérience de dégagement. Au cours de l'automne 2013, les 20 noyers dégagés et les 18 noyers témoins de l'expérience de dégagement ont été sondés afin d'évaluer la croissance avant traitements. Le prélèvement d'une carotte par arbre fut effectué à hauteur de poitrine (1,3 m). Les ouvertures formées par la sonde de Pressler ont ensuite été bouchées à l'aide de cire de greffage (Cire de greffe pour verger et pépinière, Dilmont Inc., 121 chemin Bates, Montréal, Québec, Canada) afin de limiter les risques d'infection par l'O. clavigignenti-juglandacearum. Les carottes ont été numérisées (Epson Perfection V600 Photo Scanner) à une résolution de $2400 \mathrm{PPP}$ et les cernes annuels ont été mesurés à l'aide du logiciel CooRecorder et interdatés avec CDendro (version 7,6). La croissance radiale, en $\mathrm{mm}$, pour la période de 2005 à 2010 inclusivement, avant traitement, variait de 1,03 à $7,84 \mathrm{~mm}$.

La croissance annuelle en surface terrière avant l'évaluation finale a été calculée à partir des DHP pour les noyers évalués par CIME Haut-Richelieu. Les surfaces terrières initiales $\left(\mathrm{St}_{\mathrm{i}}\right)$ et finales $\left(\mathrm{St}_{\mathrm{f}}\right)$ des 85 noyers évalués par CIME HautRichelieu ont été calculées respectivement à partir du DHP initial $\left(\mathrm{DHP}_{\mathrm{i}}\right)$ et du DHP final $\left(\mathrm{DHP}_{\mathrm{f}}\right)$ selon les formules suivantes :

$$
\begin{array}{ll}
\text { [1] } & \mathrm{St}_{\mathrm{i}}=\pi\left(\mathrm{DHP}_{\mathrm{i}} / 2\right)^{2} \\
\text { [2] } & \mathrm{St}_{\mathrm{f}}=\pi\left(\mathrm{DHP}_{\mathrm{f}} / 2\right)^{2}
\end{array}
$$

Et l'accroissement annuel en surface terrière $\left(\Delta \mathrm{St} \mathrm{t}_{\mathrm{a}}\right)$ a été calculé selon la formule suivante :

$$
\Delta \mathrm{St}_{\mathrm{a}}=\left(\mathrm{St}_{\mathrm{f}}-\mathrm{St}_{\mathrm{i}}\right) / \mathrm{A}
$$

où $\mathrm{A}$ est le nombre d'années de croissance entre les deux mesures de DHP.

La dominance est une variable ordinale alors que la croissance radiale et l'accroissement annuel en surface terrière sont des variables continues.

\section{Les variables de santé}

Les variables suivantes ont été évaluées : la mort en cime, la proportion chancrée du tronc et la vigueur. La résistance potentielle au chancre a été déduite à partir des mesures de mort en cime et de la proportion chancrée du tronc. 
La mort en cime est la mortalité des rameaux et des branches partant de l'extrémité des rameaux vers les branches puis le tronc. Elle s'évalue visuellement sur les rameaux et les branches de $2,5 \mathrm{~cm}$ ou moins. La mort en cime a été évaluée pour chaque noyer des deux dispositifs par un ou deux observateurs selon les 12 classes du projet nord-américain de l'érable (Millers et al. 1991), soit $0 \%$; 1 à $5 \%$; 6 à 15 $\%$; 16 à $25 \%$; 26 à $35 \%$; 36 à $45 \%$; 46 à $55 \%$; 56 à $65 \%$; 66 à $75 \%$; 76 à $85 \%$; 86 à $95 \%$ et 96 à $99 \%$, mais en incluant les arbres morts (100\%) dans cette dernière classe. Les chicots ne portant pas de rameaux de $2,5 \mathrm{~cm}$ de diamètre ou moins ne sont pas considérés. Les rameaux secs à l'ombre sous la cime sont présumés morts suite à l'élagage naturel et ne sont pas inclus dans l'évaluation.

La proportion chancrée du tronc s'évalue en additionnant les portions du tronc d'un noyer et de son empattement présentant des chancres, évalués en pourcentage, et regroupés selon les classes suivantes : 0 , aucun dommage; 1 , dommages sur $25 \%$ ou moins de la circonférence; 2 , dommages sur 26 à $50 \%$ de la circonférence; 3, dommages sur 51 à $75 \%$ de la circonférence; 4 , dommages sur plus de $75 \%$ de la circonférence. Cette évaluation est une modification de celle proposée par Ostry et al. (1994) qui recommandent une division en classe de $20 \%$ plutôt que $25 \%$.

La résistance potentielle est calculée selon les critères d'Ostry et al. (1994), mais en tenant compte de la modification par rapport aux dommages aux troncs. Un noyer est considéré comme potentiellement résistant (classe 1) s'il ne présente aucun dommage au tronc et moins de $50 \%$ de mort en cime ou si la proportion chancrée du tronc est de $25 \%$ ou moins et la mort en cime inférieure à $30 \%$. Dans tous les autres cas, le noyer est considéré comme sensible au chancre (classe 0).

La vigueur des noyers cendrés des deux dispositifs a aussi été mesurée selon la méthode du projet nord-américain de l'érable (Millers et al. 1991). Il s'agit d'une évaluation globale de toutes les causes de dommages sur l'ensemble de la cime, y compris les chicots, les branches mortes et les branches cassées. Les arbres sont classés comme vigoureux (classe 1) s'ils montrent moins de $10 \%$ de dommages; légèrement dépérissants (classe 2) s'ils souffrent de dommages dans 10 à $25 \%$ de la cime; modérément dépérissants (classe 3) si les dommages occupent entre 26 et $50 \%$ de la cime; gravement dépérissants (classe 4) si $51 \%$ ou plus de la cime est endommagée et morte (classe 5) si toute la cime est endommagée et sans feuillage.

La résistance potentielle est une variable binaire alors que les trois autres variables de santé sont des variables ordinales.

Selon Ostry et al. (2003), on trouve deux phénotypes d'écorce chez le noyer cendré. Le premier présente une écorce gris pâle et lisse avec des crevasses étroites et peu profondes. Le second phénotype présente une écorce rugueuse, foncée, aux crevasses profondes. Par ailleurs, nos observations nous ont aussi démontré qu'il y avait un phénotype d'écorce intermédiaire entre les deux types décrits par Ostry et al. (2003). Il s'agit ici d'une variable ordinale.

Nous avons considéré le prélèvement dans son ensemble (expérience de bouturage, voir ci-dessus), évalué à 1 pour les arbres avec prélèvement de 30 à 45 rameaux et à 0 pour les arbres sans prélèvement, comme covariable dans l'expérience de dégagement.

\section{Les statistiques}

Les variables de santé de l'expérience de dégagement ont été analysées par analyse de régression logistique séquentielle (proc LOGISTIC de SAS) en utilisant une probabilité d'entrée dans le modèle de 0,10 et de 0,055 pour le retrait de variables déjà entrées dans le modèle (Stokes et al. 1995). Les quatre variables de santé ont été utilisées individuellement comme variables dépendantes $\left(\mathrm{V}_{\mathrm{d}}\right)$ alors que le site (SaintAugustin, mont Saint-Bruno) et le traitement (dégagé ou témoin), ont été utilisés comme variables indépendantes. Le prélèvement de rameaux a été utilisé comme covariable. Les interactions de ces variables deux à deux ont été incluses dans le modèle qui se présente comme suit :

$\mathrm{V}_{\mathrm{d}}=$ Site Traitement Prélèvement Site ${ }^{\star}$ Traitement Site ${ }^{\star}$ Prélèvement Traitement ${ }^{\star}$ Prélèvement

L'adéquation du modèle a été constatée à l'aide du test du Khi carré résiduel pour lequel une probabilité supérieure à 0,05 indique un modèle adéquat (Stokes et al. 1995). L'effet des variables indépendantes significatives sur chacune des variables dépendantes a été quantifié au moyen du rapport de cote (SAS Institute 2011). Ce rapport permet de comparer l'impact de chaque variable indépendante sur la variable dépendante en évaluant la probabilité, plus grande ou moins grande, qu'un noyer soit évalué dans une classe inférieure de la variable dépendante ordinale si le niveau (variable quantitative) ou le rang (variable ordinale) de la variable indépendante est augmenté d'un niveau ou d'un rang. Lorsque les traitements de dégagement n'étaient pas significatifs, une analyse logistique séquentielle alternative a été réalisée en utilisant le phénotype et la croissance radiale initiale comme variables indépendantes d'une part, et le site et le prélèvement comme covariables d'autre part. Les seuils d'entrée et de sortie du modèle sont demeurés les mêmes, soit 0,10 et 0,055 .

De la même manière, les variables de santé des noyers cendrés évalués par les équipes de CIME Haut-Richelieu ont aussi été soumises à une analyse de régression logistique séquentielle avec les mêmes seuils pour l'entrée et l'élimination des variables indépendantes (Stokes et al. 1995) que pour l'expérience de dégagement. L'étage, l'accroissement annuel en surface terrière, le phénotype et les interactions de premier ordre entre ces trois variables ont été utilisés comme variables indépendantes. L'adéquation du modèle et l'effet des variables indépendantes significatives sur chacune des variables dépendantes ont été mesurés comme précédemment. Pour les trois interactions significatives avec le phénotype, l'analyse de régression logistique a été reprise, de façon non séquentielle, par phénotype.

Afin de vérifier l'hypothèse de Boraks et Broders (2014) concernant le diamètre plus petit des noyers à écorce lisse, une analyse de variance du DHP final des noyers évalués par CIME Haut-Richelieu a été réalisée au moyen de la procédure GLM de SAS (SAS Institute 2008), après transformation du DHP par sa racine carrée selon l'équation suivante :

$$
\sqrt{ }\left(\mathrm{DHP}_{\mathrm{f}}\right)=\text { Phénotype }
$$

Cette transformation du DHP a été effectuée pour assurer sa normalité. Des comparaisons orthogonales ont aussi été réalisées entre les différents phénotypes. 
Finalement, une analyse de variance de l'accroissement annuel en surface terrière $\left(\Delta \mathrm{St}_{\mathrm{a}}\right)$ des noyers évalués par CIME Haut-Richelieu a été réalisée au moyen de la procédure GLM de SAS (SAS Institute 2008), après transformation de l'accroissement annuel, selon les équations suivantes :

$$
\begin{aligned}
& \text { [6] } \sqrt{ }\left(\Delta \mathrm{St}_{\mathrm{a}}\right)=\text { Étage Phénotype Étage }{ }^{\star} \text { Phénotype } \\
& \text { [7] } \sqrt{ }\left(\Delta \mathrm{St}_{\mathrm{a}}\right)=\text { Étage Phénotype }
\end{aligned}
$$

La transformation de l'accroissement annuel par sa racine carrée a été effectuée pour assurer sa normalité. La moyenne des moindres carrés de la variable dépendante a été calculée pour chaque valeur de l'étage et du phénotype. Des comparaisons orthogonales ont aussi été réalisées entre les différents étages et les différents phénotypes.

\section{Résultats et discussion \\ L'expérience de dégagement}

Les résultats de l'analyse de régression logistique séquentielle des variables de santé sont présentés au Tableau 3. Aucun effet significatif n'a été détecté pour le dégagement des noyers, quelle que soit la variable de santé examinée. Ces résultats concordent avec ceux de Tanguay et al. (2018), qui, pour leur part, n’ont pas trouvé de différences significatives entre la production de conidies des noyers dégagés en comparaison des noyers témoins, dans ce même dispositif. Les modèles logistiques pour la vigueur et la résistance sont adéquats alors que la faible probabilité pour le Khi carré des résidus de la mort en cime démontre que le modèle trouvé est incomplet. Aucune variable indépendante n'avait d'effet significatif sur les dommages au tronc. Le site a une influence significative sur la mort en cime et sur la vigueur des noyers. Ainsi, un noyer cendré poussant à la Station agronomique de l'Université Laval a cinq fois moins de chance de présenter une mort en cime élevée par rapport à celui se développant au mont Saint-Bruno (rapport de cote 5,2, Tableau 3). De fait, 79 $\%$ des noyers, de la Station agronomique, dégagés ou non, ont $20 \%$ ou moins de mort en cime contre seulement $48 \%$ pour ceux du mont Saint-Bruno. Pareillement, ces noyers de la Station agronomique ont aussi 13 fois plus de chance d'être évalués comme vigoureux (vigueur 1) que s'ils poussaient au mont Saint-Bruno (rapport de cote 12,6, Tableau 3), de sorte que $63 \%$ des noyers à Saint-Augustin sont vigoureux, contre $21 \%$ pour le mont Saint-Bruno. Les travaux de Sambaraju et al. (2018) ont déjà démontré que les noyers localisés au sudouest de la province présentaient plus de dommages au tronc que ceux localisés plus au nord et à l'est. Ces dommages ont

Tableau 3. Analyse de régression logistique séquentielle des variables de santé de l'expérience de mise en lumière de noyers cen-

\begin{tabular}{|c|c|c|c|c|}
\hline \multicolumn{2}{|c|}{ Variables } & \multirow[b]{2}{*}{ Probabilité } & \multirow[b]{2}{*}{ Rapport de cote $\mathrm{e}^{\mathrm{a}}$} & \multirow{2}{*}{$\begin{array}{c}\text { Probabilité du } \\
\text { Khi carré résiduel }\end{array}$} \\
\hline Dépendante & Indépendante & & & \\
\hline \multirow[t]{6}{*}{ Dommage au tronc } & Dégagement & 0,40 & - & 0,67 \\
\hline & Site & 0,55 & - & - \\
\hline & Prélèvements & 0,33 & - & - \\
\hline & Dégagement x Site & 0,30 & - & - \\
\hline & Dégagement x Prélèvements & 0,78 & - & - \\
\hline & Site x Prélèvements & 0,25 & - & - \\
\hline \multirow[t]{6}{*}{ Mort en cime } & Dégagement & 0,18 & - & 0,038 \\
\hline & Site & 0,0082 & 5,2 & - \\
\hline & Prélèvements & 0,09 & - & - \\
\hline & Dégagement x Site & 0,71 & - & - \\
\hline & Dégagement x Prélèvements & 0,48 & - & - \\
\hline & Site x Prélèvements & 0,29 & - & - \\
\hline \multirow[t]{6}{*}{ Vigueur } & Dégagement & 0,46 & - & 0,12 \\
\hline & Site & 0,0009 & 12,6 & - \\
\hline & Prélèvements & 0,0316 & 4,2 & - \\
\hline & Dégagement x Site & 0,99 & - & - \\
\hline & Dégagement x Prélèvements & 0,76 & - & - \\
\hline & Site x Prélèvements & 0,12 & - & - \\
\hline \multirow[t]{6}{*}{ Résistance } & Dégagement & 0,38 & - & 0,48 \\
\hline & Site & 0,36 & - & - \\
\hline & Prélèvements & 0,0271 & 0,16 & - \\
\hline & Dégagement x Site & 0,28 & - & - \\
\hline & Dégagement x Prélèvements & 0,60 & - & - \\
\hline & Site x Prélèvements & 0,84 & - & - \\
\hline
\end{tabular}
drés au Mont Saint-Bruno et à la Station agronomique de l'Université Laval, en fonction du dégagement

${ }^{a}$ Les rapports de cote ne sont donnés que pour les variables indépendantes significatives $(\mathrm{P} \leq 0,05)$. Un noyer cendré a cinq $(5,2)$ fois plus de chance de se trouver dans une classe de mort en cime inférieure (ex. $20 \%$ ou moins) s'il pousse à la Station Agronomique de l'Université Laval plutôt qu'au Mont Saint-Bruno. Pareillement, un noyer de la Station Agronomique a $13(12,6)$ fois plus de chances d'être vigoureux que s'il poussait au Mont Saint-Bruno. Les noyers auront aussi quatre (4,2) fois plus de chance d'être évalués comme vigoureux si on a prélevé des rameaux dans leur cime. Ces mêmes noyers ont six fois moins $(0,16)$ de chances d'être considérés sensibles au chancre. 
une influence sur l'évaluation de la résistance au chancre. Ces mêmes travaux ont aussi démontré que les noyers poussant en altitude présentent plus de mort en cime. Le site du mont Saint-Bruno est localisé vers le sud-ouest par rapport à celui de la Station agronomique et son élévation est supérieure.

Les noyers sur lesquels des rameaux ont été prélevés ont cinq fois plus de chances (Tableau 3) d'être classés comme vigoureux (vigueur, classe 1) que ceux sur lesquels aucun prélèvement n'a été fait. Pareillement, ils ont aussi six fois moins de chances (Tableau 3) d'être évalués comme sensibles (résistance potentielle, classe 0) au chancre. Compte tenu de la sélection au hasard des noyers ayant subi un prélèvement, les caractéristiques particulières de ces noyers en début d'expérience peuvent difficilement être évoquées pour expliquer l'impact des prélèvements de rameaux. Nos résultats suggèrent plutôt que ceux-ci ont stimulé les réactions physiologiques des noyers ainsi traités. Rioux et al. (2018) ont notamment démontré que des blessures mécaniques, même sans inoculation d'agent pathogène, pouvaient provoquer l'apparition de zones de réaction dans le xylème, ces dernières s'imprégnant de phénols de la même manière que chez les noyers inoculés et que, par la suite, ces zones se couvraient d'une nouvelle écorce. D’autre part, Clark (1955) a montré que l'élagage de $25 \%$ ou $50 \%$ des rameaux de noyers noirs (Juglans nigra L.) augmentait à la fois sa croissance en diamètre et en hauteur, ce qui, en termes de santé des arbres, peut être synonyme de résistance accrue aux ravageurs. Par ailleurs, Jovellar Lacambra et al. (2012) ont rapporté que des chênes verts (Quercus ilex L.) ayant subi un élagage de branches basses en jeune âge montraient significativement moins de mort en cime que ceux n'ayant pas été élagués. S’il existe une relation de cause à effet entre le prélèvement de rameaux, l'élimination du bois mort et la meilleure santé des noyers, alors l'élagage et la taille de formation des cimes des noyers, pour l'obtention de bois de qualité (Hubert et Courraud 1994) ou même pour des raisons esthétiques, pourraient contribuer à en améliorer la santé, à condition de respecter les bonnes pratiques en la matière (Thibault 1993; Bureau de normalisation du Québec 2020).

Les résultats de l'analyse statistique alternative (régression logistique) pour l'expérience de dégagement sont présentés au Tableau 4. Comme pour l'analyse initiale, aucun effet significatif des facteurs considérés n'a été trouvé pour les dommages au tronc alors que les effets sur la résistance au chancre demeurent les mêmes. Pour la mort en cime et la vigueur, l'effet du site demeure sensiblement le même mais la croissance radiale des noyers avant le dégagement des cimes influence significativement ces deux variables de santé. Ainsi, pour chaque augmentation théorique de $2 \mathrm{~mm}$ de croissance radiale de 2005 à 2010, les noyers ont une probabilité trois fois plus grande de présenter une mort en cime évaluée dans la classe inférieure à celle des noyers n'ayant pas cette augmentation. Pareillement, la vigueur de ces noyers à croissance théorique supérieure aura trois fois plus de chances d'être évaluée dans une meilleure classe. Tous les modèles sont adéquats.

\section{Les données de santé recueillies par CIME Haut-Richelieu}

Finalement, l'analyse des données de santé des noyers cendrés recueillies par CIME Haut-Richelieu (Tableau 5) montre que l'étage dans lequel se trouvent ces noyers a un impact significatif sur les dommages au tronc et sur la résistance

Tableau 4. Analyse de régression logistique séquentielle des variables de santé de l'expérience de mise en lumière de noyers cendrés au Mont Saint-Bruno et à la Station agronomique de l'Université Laval, en fonction des autres variables

\begin{tabular}{|c|c|c|c|c|}
\hline \multicolumn{2}{|c|}{ Variables } & \multirow[b]{2}{*}{ Probabilité } & \multirow[b]{2}{*}{ Rapport de cote ${ }^{a}$} & \multirow{2}{*}{$\begin{array}{l}\text { Probabilité du } \\
\text { Khi carré résiduel }\end{array}$} \\
\hline Dépendante & Indépendante & & & \\
\hline \multirow{4}{*}{ Dommages au tronc } & Site & 0,55 & - & 0,84 \\
\hline & Prélèvements & 0,33 & - & - \\
\hline & Croissance radiale ${ }^{\mathrm{b}}$ & 0,91 & - & - \\
\hline & Phénotype & 0,71 & - & - \\
\hline \multirow{4}{*}{ Mort en cime } & Site & 0,0085 & 5,2 & 0,25 \\
\hline & Prélèvements & 0,23 & - & - \\
\hline & Croissance radiale ${ }^{b}$ & 0,0017 & $3,5^{\mathrm{c}}$ & - \\
\hline & Phénotype & 0,22 & - & - \\
\hline \multirow[t]{4}{*}{ Vigueur } & Site & 0,0009 & 10,9 & 0,17 \\
\hline & Prélèvements & 0,084 & - & - \\
\hline & Croissance radiale ${ }^{b}$ & 0,0053 & $3,3^{c}$ & - \\
\hline & Phénotype & 0,44 & - & - \\
\hline \multirow[t]{4}{*}{ Résistance } & Site & 0,36 & - & 0,21 \\
\hline & Prélèvements & 0,0271 & 0,16 & - \\
\hline & Croissance radiale ${ }^{b}$ & 0,0684 & - & - \\
\hline & Phénotype & 0,97 & - & - \\
\hline
\end{tabular}

${ }^{a}$ Les rapports de cote ne sont donnés que pour les variables indépendantes significatives $(\mathrm{P} \leq 0,05)$.

${ }^{\mathrm{b}}$ Croissance radiale avant dégagement, de 2005 à 2010.

'Pour une croissance radiale de deux mm. Ainsi, un groupe de noyers présentant une croissance radiale périodique supérieure de $2 \mathrm{~mm}$ à un autre groupe, de croissance plus faible, aura cinq fois plus de chance d'être classé dans une classe de mort en cime faible (ex $20 \%$ et moins) et d'être considéré comme vigoureux (classe 1) 
Tableau 5. Analyse de régression logistique séquentielle des variables de santé des noyers cendrés recueillies par CIME HautRichelieu

\begin{tabular}{|c|c|c|c|c|}
\hline \multicolumn{2}{|c|}{ Variables } & \multirow[b]{2}{*}{ Probabilité } & \multirow[b]{2}{*}{ Rapport de cote ${ }^{a}$} & \multirow{2}{*}{$\begin{array}{l}\text { Probabilité du } \\
\text { Khi carré résidue }\end{array}$} \\
\hline Dépendante & Indépendante & & & \\
\hline \multirow[t]{6}{*}{ Dommages au tronc } & Croissance $^{\mathrm{b}}$ & 0,50 & - & 0,78 \\
\hline & Étage & 0,0027 & 0,47 & - \\
\hline & Phénotype & 0,55 & - & - \\
\hline & Croissance x Étage & 0,78 & - & - \\
\hline & Croissance x Phénotype & 0,61 & - & - \\
\hline & Étage x Phénotype & 0,47 & - & - \\
\hline \multirow[t]{6}{*}{ Mort en cime } & Croissance $^{\mathrm{b}}$ & 0,0001 & $14,7^{\mathrm{c}}$ & 0,81 \\
\hline & Étage & 0,80 & - & - \\
\hline & Phénotype & 0,0218 & 3,5 & - \\
\hline & Croissance x Étage & 0,89 & - & - \\
\hline & Croissance x Phénotype & 0,0322 & - & - \\
\hline & Étage x Phénotype & 0,56 & - & - \\
\hline \multirow[t]{6}{*}{ Vigueur } & Croissance $^{\mathrm{b}}$ & 0,0021 & $2,3^{c}$ & 0,04 \\
\hline & Étage & 0,28 & - & - \\
\hline & Phénotype & 0,23 & - & - \\
\hline & Croissance x Étage & 0,13 & - & - \\
\hline & Croissance x Phénotype & 0,028 & - & - \\
\hline & Étage x Phénotype & 0,036 & - & - \\
\hline \multirow[t]{6}{*}{ Résistance } & Croissance $^{\mathrm{b}}$ & 0,40 & - & 0,26 \\
\hline & Étage & 0,0283 & 1,95 & - \\
\hline & Phénotype & 0,46 & - & - \\
\hline & Croissance x Étage & 0,80 & - & - \\
\hline & Croissance x Phénotype & 0,62 & - & - \\
\hline & Étage x Phénotype & 0,29 & - & - \\
\hline
\end{tabular}

${ }^{a}$ Les rapports de cote ne sont donnés que pour les variables indépendantes significatives $(\mathrm{P} \leq 0,05)$. La probabilité qu’un noyer soit dans la classe de dommage au tronc la plus faible diminue de $50 \%(0,47)$ et probabilité d'être sensible au chancre (résistance 0$)$ augmente du double $(1,95)$ lorsque l'arbre est situé un étage plus bas dans la canopée. Pour chaque augmentation de classe de phénotype d'écorce lisse vers rugueux, la chance qu'un noyer soit dans une classe de mort en cime plus faible augmente de 3,5 fois.

${ }^{\mathrm{b}} \mathrm{La}$ croissance annuelle en surface terrière, calculée sur un période variant de 2 à 7 ans, selon les noyers évalués.

cPour une croissance annuelle en surface terrière de de $20 \mathrm{~cm}^{2}$. Compte-tenu de l'interaction significative entre la croissance et le phénotype pour la mort en cime et la vigueur, ces variables sont analysées au tableau 6 .

potentielle au chancre. Ainsi, la probabilité qu'un noyer ait des dommages au tronc sur moins de $25 \%$ de sa circonférence diminue de moitié chaque fois qu'on descend d'un étage dans le couvert forestier, de sorte que $53 \%$ des noyers dominants ont $25 \%$ ou moins de dommages au tronc, alors que $67 \%$ des noyers opprimés ont plus de $75 \%$ de la circonférence du tronc affectée par des chancres. De même, la probabilité d'être sensible au chancre augmente presque du double à chaque fois qu'on diminue d'un étage. Dans l'étage dominant, $58 \%$ des noyers sont sensibles au chancre alors que $42 \%$ présentent de la résistance potentielle. Par ailleurs, $83 \%$ des noyers opprimés sont sensibles alors que seulement $17 \%$ montrent de la résistance.

Le phénotype et la croissance annuelle en surface terrière ont une influence significative sur la mort en cime des noyers. Pour chaque augmentation de classe de phénotype d'écorce allant de lisse à rugueux, la probabilité qu'un noyer soit dans une classe de mort en cime plus faible augmente de 3,5 fois. L'interaction significative entre le phénotype et la croissance exige que cette dernière soit examinée pour chaque phénotype d'écorce (Tableau 5). La croissance annuelle a un effet significatif sur la mort en cime pour le phénotype à écorce lisse $(\mathrm{P}=0,0045$, Tableau 6) et, pour chaque augmentation de $20 \mathrm{~cm}^{2}$ de croissance, la probabilité d'un noyer de se trouver dans la classe de mort en cime la plus faible augmente de 16 fois. La croissance annuelle en surface terrière n'a pas d'effet significatif sur la mort en cime des noyers dont le phénotype d'écorce est intermédiaire $(\mathrm{P}=0,10)$ ou rugueux $(\mathrm{P}=0,23)$ (Tableau 6). Pour leur part, Sambaraju et al. (2018) ont démontré qu'il existait une différence significative de la mort en cime des noyers cendrés entre les phénotypes d'écorce ainsi qu'entre les noyers dominants ou poussant à découvert en comparaison aux noyers intermédiaires et opprimés.

La croissance annuelle en surface terrière a un effet significatif sur la vigueur ( $\mathrm{P}=0,0021$; Tableau 5). L'interaction entre la croissance et le phénotype d'une part et entre l'étage et le phénotype d'autre part exigent que ces analyses soient refaites pour chaque phénotype. La croissance annuelle en surface terrière n'a d'effet significatif que pour les noyers à écorce lisse et pâle ( $\mathrm{P}=0,0427$, Tableau 6). Ainsi, pour chaque augmentation annuelle de $20 \mathrm{~cm}^{2}$ en surface terrière, un individu a 16 fois plus de chance de se trouver dans la meilleure classe de vigueur que l'arbre ayant une croissance inférieure. Les probabilités décroissantes de l'impact de l'étage, des 
Tableau 6. Analyse de régression logistique des variables mort en cime et vigueur des noyers cendrés recueillies par CIME HautRichelieu en fonction du phénotype

\begin{tabular}{|c|c|c|c|c|}
\hline \multicolumn{2}{|c|}{ Variables } & \multirow{2}{*}{$\begin{array}{l}\text { Phénotype } \\
\text { d'écorce }\end{array}$} & \multirow[b]{2}{*}{ Probabilité } & \multirow{2}{*}{$\begin{array}{l}\text { Rapport } \\
\text { de cote }^{\mathrm{a}}\end{array}$} \\
\hline Dépendante & Indépendante & & & \\
\hline \multirow[t]{3}{*}{ Mort en cime } & \multirow[t]{3}{*}{ Croissance $^{\mathrm{b}}$} & Lisse et pâle & 0,0045 & $15,8^{\mathrm{c}}$ \\
\hline & & Intermédiaire & 0,10 & - \\
\hline & & Foncé et rugueux & 0,23 & - \\
\hline \multirow[t]{3}{*}{ Vigueur } & \multirow[t]{3}{*}{ Croissance $^{b}$} & Lisse et pâle & 0,0427 & $15,7^{\mathrm{c}}$ \\
\hline & & Intermédiaire & 0,07 & - \\
\hline & & Foncé et rugueux & 0,20 & - \\
\hline \multirow[t]{3}{*}{ Vigueur } & \multirow[t]{3}{*}{ Étage $^{\mathrm{d}}$} & Lisse et pâle & 0,68 & - \\
\hline & & Intermédiaire & 0,26 & - \\
\hline & & Foncé et rugueux & 0,12 & - \\
\hline
\end{tabular}

aLes rapports de cote ne sont donnés que pour les variables indépendantes significatives $(\mathrm{P} \leq 0,05)$.

bLa croissance annuelle en surface terrière, calculée sur un période variant de 2 à 7 ans, selon les noyers évalués.

'Pour une croissance annuelle en surface terrière de de $20 \mathrm{~cm}^{2}$. La croissance annuelle a un effet significatif sur la mort en cime seulement pour le phénotype à écorce lisse et, pour chaque augmentation de $20 \mathrm{~cm}^{2}$ de croissance, la probabilité d'un noyer de se trouver dans la classe de mort en cime la plus faible augmente de 16 fois. On constate le même effet pour la vigueur.

dL'effet de l'étage sur la vigueur tend à être plus important chez les noyers à écorce foncée et rugueuse, sans que cela soit significatif statistiquement

noyers à écorce lisse vers les noyers à écorce rugueuse, suggèrent que l'étage pourrait avoir une influence pour ces derniers. Cependant, la faible probabilité du Khi carré résiduel (Tableau 5) indique que d'autres facteurs influenceraient aussi la vigueur.

Le diamètre à hauteur de poitrine et l'accroissement annuel en surface terrière

L'analyse de variance $(\mathrm{P}=0,0049)$ et les comparaisons orthogonales montrent que le diamètre transformé des noyers à écorce lisse ou intermédiaire est significativement plus petit que celui des noyers à écorce foncée et rugueuse (Tableau 7). Ces résultats confirment ceux de Boraks et Broders (2014).

Par ailleurs, l'étage $(\mathrm{P}<0,0001)$ et le phénotype d'écorce $(\mathrm{P}=0,0087)$ ont une influence significative sur l'accroissement annuel en surface terrière transformé. L'interaction entre ces deux variables n'est pas significative $(P=0,80)$. Les moyennes des moindres carrés de l'accroissement annuel en surface terrière transformé, $\sqrt{ }\left(\Delta S t_{2}\right)$, et les moyennes de cet accroissement, $\Delta \mathrm{St}_{\mathrm{a}}$, en fonction de l'étage et du phénotype d'écorce sont présentées au Tableau 8. Plus les noyers occupent une position dominante, plus leur croissance est élevée. Les différences de croissance entre les étages sont toutes significatives (Tableau 8).

Avec une croissance supérieure, la mort en cime est faible et la vigueur est bonne. L'augmentation de la lumière sur les noyers cendrés suite au détourage de cime n'a pas amélioré significativement la santé des noyers traités. Il est possible que le prélèvement de rameaux et l'élimination de bois mort, réalisés principalement sur des noyers témoins, aient masqué l'effet du détourage de cime. L'étage a un effet significatif sur certaines variables de santé. Les données de l'expérience de dégagement (détourage) et celles de CIME Haut-Richelieu suggèrent que la croissance soit responsable de la meilleure santé des noyers. Elle serait aussi responsable de la position dominante de certains noyers dans le couvert forestier.
Tableau 7. Moyennes des moindres carrés de la racine carrée du diamètre final, $\sqrt{ }\left[\mathrm{DHP}_{\mathrm{f}}\right]$, et moyennes de ces $\mathrm{DHP}_{\mathrm{f}}$, en fonction du phénotype d'écorce pour les données soumises par CIME Haut-Richelieu

\begin{tabular}{lcc}
\hline Phénotype & $\begin{array}{c}\text { Moyenne des moindres } \\
\text { carrés }^{\mathbf{a}} \mathbf{d e} \sqrt{\left(\mathbf{D H P}_{\mathrm{f}}\right)}\end{array}$ & $\begin{array}{c}\text { Moyenne } \mathrm{DHP}_{\mathrm{f}} \\
(\mathbf{c m})\end{array}$ \\
\hline Lisse et pâle & $4,3 \mathrm{~b}$ & 20,1 \\
Intermédiaire & $4,1 \mathrm{~b}$ & 18,7 \\
Foncé et rugueux & $5,4 \mathrm{a}$ & 32,2 \\
\hline
\end{tabular}

${ }^{a}$ Les moyennes des moindres carrés suivies par une lettre différente sont significativement différentes d'après les comparaisons orthogonales réalisées sur ces variables $(\mathrm{P} \leq 0,05)$

Tableau 8. Moyennes des moindres carrés de la racine carrée de l'accroissement annuel en surface terrière, $\sqrt{ }\left[\Delta \mathbf{S t}_{\mathrm{a}}\right]$, et moyennes de cet accroissement, $\Delta \mathbf{S t}_{\mathrm{a}}$, en fonction de l'étage et du phénotype d'écorce, pour les données soumises par CIME Haut-Richelieu

\begin{tabular}{llcc}
\hline Variable & Catégorie & $\begin{array}{c}\text { Moyenne des moindres } \\
\text { carrés }^{\mathbf{a}} \sqrt{\left(\Delta \mathbf{S t}_{\mathbf{a}}\right)}\end{array}$ & $\begin{array}{c}\text { Moyenne } \Delta \mathbf{S t}_{\mathbf{a}} \\
\left(\mathbf{c m}^{2}\right)\end{array}$ \\
\hline Étage & dominant & $5,2 \mathrm{a}$ & 29,7 \\
& codominant & $3,6 \mathrm{~b}$ & 15,7 \\
& intermédiaire & $2,4 \mathrm{c}$ & 5,9 \\
& opprimé & $1,0 \mathrm{~d}$ & 0,6 \\
\multirow{2}{*}{ Phénotype } & & \\
& lisse & $2,4 \mathrm{~b}$ & 8,5 \\
& intermédiaire & $2,9 \mathrm{~b}$ & 13,2 \\
& rugueux & $3,8 \mathrm{a}$ & 25,8 \\
\hline
\end{tabular}

aPour chaque variable, les moyennes des moindres carrés suivies par une lettre différente sont significativement différentes d'après les comparaisons orthogonales réalisées sur ces variables $(\mathrm{P} \leq 0,05)$ 
Les noyers cendrés à écorce rugueuse ont un DHP supérieur et une meilleure croissance annuelle que ceux à écorce lisse ou intermédiaire. Nous avons démontré l'impact du phénotype d'écorce foncé et rugueux sur au moins une variable de santé. Mais il est probable que cet impact soit lié à la croissance significativement plus élevée de ces noyers (Tableau 8). Des analyses moléculaires seraient nécessaires pour vérifier si des gènes spécifiques déterminent la présence de cette écorce foncée et profondément crevassée qu'on retrouve même chez les noyers cendrés de petit diamètre (Tableau 2) et pour déterminer si ces gènes sont aussi liés à la résistance au chancre.

Ainsi, nos résultats suggèrent que dans le cadre d'un programme de sélection de noyers cendrés résistants au chancre causé par l'O. clavigignenti-juglandacearum (Environnement Canada 2010), il convient de privilégier les individus ayant une croissance supérieure. L'étage dominant et le phénotype d'écorce rugueux sont de bons indicateurs de la croissance supérieure d'un noyer cendré.

Il serait souhaitable qu'une expérience comparant des noyers cendrés dégagés, d'autres élagués et d'autres sans intervention, soit réalisée afin d'éclaircir l'impact de ces deux pratiques sylvicoles sur la santé et la survie des noyers. Mohni et al. (2009) rapportent que le noyer commun (Juglans regia L.) en plantations dont le couvert est fermé réagit lentement lorsqu'il est dégagé. Stringer et Wittwer (1985) ont montré que le noyer noir réagissait jusqu'à 12 ans après dégagement. Un suivi à plus long terme des noyers de l'expérience de dégagement serait donc souhaitable, comme un délai de réaction après dégagement peut être supérieur à cinq ans.

\section{Remerciements}

Les auteurs remercient Hugues Sansregret, Charles Villeneuve, André Lapierre et Julie Bouliane de l'Université Laval, Robert Werbiski de la Défense nationale et Valérie Deschenes et Renée Gagnon de l'organisme CIME Haut-Richelieu pour leur soutien dans le cadre de ces travaux. Nous remercions aussi Martine Blais et Caroline Bourdon du Service canadien des forêts de Ressources naturelles Canada pour la prise des données de santé dans le cadre de ce projet et Lauriane Monette, Valérie Deschêsnes, Marie-France Côté, Patrick Laniel, Maxime Tremblay, Camille PelletierGuittier, Laurence Teyssier, Yolande Boyer, Diane Ravit, Maxime Quesnel, Marie-Pier Prairie, Élise Carpentier, Marie-Pier Lavallée, Pierre Jackson et Étienne Drouin pour le suivi des noyers des sites de CIME Haut-Richelieu.

Nos remerciements vont aussi au Fonds interministériel pour le rétablissement d'Environnement Canada et au ministère de la Défense nationale pour avoir financé ce projet, ainsi qu'à la fondation Rêverie, pour la bourse d'études octroyée à Nicolas Nadeau-Thibodeau.

\section{Références}

Abgrall, J.F. et A. Soutrenon. 1991. La forêt et ses ennemis, $3^{\text {ème }}$ édition. Grenoble, CEMAGREF, pp. 147-150, 247-250, 377-380.

Amorini, E., M.C. Manetti, T. Turchetti, A. Sansotta et F. Villani. 2001. Impact of silvicultural system on Cryphonectria parasitica incidence and on genetic variability in a chestnut coppice in Central Italy. For. Ecol. Manag. 142: 19-31.

Boraks, A. et K.D. Broders. 2014. Butternut (Juglans cinerea) health, hybridization, and recruitment in the northeastern United
States Can. J. For. Res. 44: 1244-1252. dx.doi.org/10.1139/cjfr-20140166

Broders, K.D. et G.J. Boland. 2011. Reclassification of the butternut canker fungus, Sirococcus clavigignenti-juglandacearum into the genus Ophiognomonia. Fungal Biol. 115: 70-79. doi:10.1016/j.funbio.2010.10.007

Broders, K.D., A. Boraks, A.M. Sanchez et G.J. Boland. 2012. Population structure of the butternut fungus, Ophiognomonia clavigignenti-juglandacearum, in North American forests. Ecol. Evol. 2: 2114-2127.

Bureau de normalisation du Québec. 2020. Norme NQ 0605200/2001 Entretien arboricole et horticole, $2^{\mathrm{e}}$ édition révisée. SainteFoy, Québec, pp. 15-30, 81-97.

Clark, F.B. 1955. Black walnut responds to pruning. J. For. 53: 362365.

Corneil, J.A. et L.F. Wilson. 1979. Life history of the butternut curculio, Conotrachelus juglandis (Coleoptera: Curculionidae), in Michigan. Great Lakes Entomol. 12: 13-15.

Davis, C.N., D.T. Myren et E.J. Czerwinski. 1992. First report of butternut canker in Ontario. Plant Dis. 76: 972

D'Eon, S.P., L.P. Magasi, D. Lachance et P. DesRochers. 1995. DNARPA, Réseau national de surveillance de l'état de santé des forêts au Canada : Guide d'établissement et de surveillance des parcelles (version revue). Rapport d'information Pi-X-117F, Chalk River, Ontario, 107 pp.

DesRochers, P., D. Cloutier, J. Bérubé, M. Blais, K. Savard et J. Thibault. 2010. État de santé du noyer cendré (Juglans cinerea L.) sur les terres fédérales au Québec en 2006-2008. Ressources naturelles Canada, Service canadien des forêts, Centre de foresterie des Laurentides, Rapport interne, 70 pp.

Environnement Canada. 2010. Programme de rétablissement du noyer cendré (Juglans cinerea) au Canada. Série de Programmes de rétablissement de la Loi sur les espèces en péril, Environnement Canada, Ottawa, vii + 29 pp.

Farrar, J.L. 1996. Les arbres du Canada. Fides, Saint-Laurent (QC) et Service canadien des forêts, Ottawa Ontario, 502 pp.

Farris, M. et J. Appleby. 1979. The biology of the walnut caterpillar, Datana integerrima. In : Proceedings of the North Central Branch, Entomological Society of America, 33: 50.

Furnier, G.R., A.M. Stolz, R.M. Mustaphi et M.E. Ostry. 1999. Genetic evidence that butternut canker was recently introduced into North America. Can. J. Bot. 77: 783-785.

Grandtner, M.M. 1966. La végétation forestière du Québec méridional - 7 tirage, 1981. Québec, LePresses de l'Université Laval, 216 pp. Graves, A.H. 1923. The Melanconis disease of the Butternut (Juglans cinerea L.). Phytopathology 13: 411-434.

Harrison, K.J., J.E. Hurley et M.E. Ostry. 1998. First report of butternut canker caused by Sirococcus clavigignenti-juglandacearum in New Brunswick, Canada. Plant Dis. 82: 1282.

Hubert, M. et R. Courraud. 1994. Élagage et taille de formation des arbres forestiers, $2^{\mathrm{e}}$ édition. Institut pour le développement forestier, Paris, 303 pp.

Innes, L. et A. Rainville. 1996. Distribution et détection du Sirococcus clavigignenti-juglandacearum au Québec. Phytoprotection 77: 75-78. http://id.erudit.org/iderudit/706102ar

Jacobi, W.R., P. Pineda Bovin, K.S. Burns, A. Crump et B.A. Goodrich. 2017. Pruning limber pine to reduce impacts from white pine blister rust in the Southern Rocky Mountains. For. Sci. 63: 218-224. https://doi.org/10.5849/forsci.16-011.

Jovellar Lacambra, L.C., A. Garcia Martin et R. San Martin Fernandez. 2012. Effects of microsite conditions and early pruning on growth and health status of holm oak plantations in CentralWestern Spain. New Forests 43: 887-903. https://doi.org/ 10.1007/s11056-012-9335-7

Kuntz, J.E., A.J. Prey, S. Jutte et V.M.G. Nair. 1979. The etiology, distribution, epidemiology, histology and impacts of butternut cancer in Wisconsin. In : Walnut insects and diseases. Workshop pro- 
ceedings, June 13-14, 1978. Gen. Tech. Rept. NC-52, USDA For. Serv., pp. 69-72.

Laflamme, G. 1999. Traitement réussi d'une plantation de pins rouges affectée par le Gremmeniella abietina, race européenne. Phytoprotection 80 : 55-64. https://doi.org/10.7202/706180ar.

Lupien, P. 2006. Des feuillus nobles en Estrie et au Centre-du-Québec - Guide de mise en valeur. Association forestière des Cantons de l'Est, Sherbrooke, pp. 24-25 et 116-120.

Lupien, P. 2013. Le noyer cendré. In : Ministère des Ressources Naturelles. Le guide sylvicole du Québec, Tome 1, Les fondements biologiques de la sylviculture, ouvrage collectif sous la supervision de B. Boulet et M. Huot, Les Publications du Québec, pp. 68-69.

Majcen, Z. 1995. Le noyer cendré au lac Tapani. Note de recherche forestière no 64. Direction de la recherche forestière, ministère des Ressources naturelles, Sainte-Foy (Québec). 5 pp.

McIlwrick, K., S. Wetzel, T. Beardmore et K. Forbes. 2000. Ex situ conservation of American chestnut (Castanea dentata (Marsh.) Borkh.) and butternut (Juglans cinerea L.) : A review. For. Chron. 76: 765-774.

Michler, C.H., P.M. Pijut, D.F. Jacobs, R. Meilan, K.E. Woeste et M.E. Ostry. 2006. Improving disease resistance of butternut (Juglans cinerea), a threatened fine hardwood: A case for single-tree selection through genetic improvement and deployment. Tree Physiol. 26: 121-128.

Millers, I., D. Lachance, W.G. Burkman et D.C. Allen. 1991. North American sugar maple decline project: organization and field methods. USDA For. Serv. Northeast. For. Exp. Stn. Gen. Tech. Rep. NE-154/Forestry Canada, 26 pp.

Mohni, C., F. Pelleri et G.E. Hemery. 2009. The modern silviculture of Juglans regia L.: A literature review. Die Bodenkultur 60: 21-34. Nadeau-Thibodeau, N. 2015. Le chancre causé par l'Ophiognomonia clavigignenti-juglandacearum : protection et rétablissement du noyer cendré. Université Laval, Faculté des études supérieures et postdoctorales, Mémoire de maîtrise, $119 \mathrm{pp}$.

Nair, V.M.G., C.J. Kostichka et J.E. Kuntz. 1979. Sirococcus clavigignenti-juglandacearum: an undescribed species causing canker on butternut. Mycologia 71: 641-645.

Nielsen, C., M. Cherry, B. Boysen, A. Hopkin, J. McLaughlin et T. Beardmore. 2003. Évaluation et rapport de situation du COSEPAC sur le noyer cendré (Juglans cinerea) au Canada. Comité sur la situation des espèces en péril au Canada, Ottawa, $37 \mathrm{pp}$.

Niinemets, Ü. et F. Valladares. 2006. Tolerance to shade, drought, and waterlogging of temperate northern hemisphere trees and shrubs. Ecol. Monog. 76: 521-547.

Orchard, L.P., J.E. Kuntz et K.J. Kessler. 1982. Reactions of Juglans species to butternut canker and implications for disease resistance. In: Proceedings of the Black walnut for the Future: Gen. Tech. Rep. NC-74. U. S. Dep. Agric. For. Serv. North Central For. Exp. Stn., St. Paul, MN. pp. 27-31.

Ostry, M.E. 1997. Butternut canker: history, biology, impact, and resistance. In Knowledge for the future of black walnut. Proceedings of the 5th black walnut symposium, Sambeek, J.W. van, (ed.), July 28 31, 1996, Springfield, MO, USDA For. Serv. NC-191, pp. 192-199.

Ostry, M.E. et K. Woeste. 2004. Spread of butternut canker in North America, host range, evidence of resistance within butternut populations and conservation genetics. In : Black Walnut in a new century. Proceedings of the $6^{\text {th }}$ Walnut Council research symposium, Michler, C.H., Pijut, P.M., Sambeek, J.W., van, Coggeshall, M.V., Seifert, J., Woeste, K., Overton, R. and Ponder, F., Jr. (eds), July 25-28, Lafayette, IN. Gen. Tech. Rep., St. Paul, MN, USDA For. Serv. NC-243, pp. 37-44.
Ostry, M.E., M. Mielke et D. Skilling. 1994. Butternut - strategies for managing a threatened tree. Gen. Tech. Rep. NC-165. St. Paul, MN: USDA Forest Service, North Central Forest Experimental Station, $7 \mathrm{pp}$.

Ostry, M. E., B. Ellingson, D. Seekins et W. Ruckheim. 2003. The need for silvicultural practices and collection of butternut germplasm for species conservation. In: Proceedings of the 13th Central Hardwood Forest Conference. J. W. Van Sambeek, J. O. Dawson, F. Ponder, Jr., E. F. Loewenstein and J. S. Fralish (Eds.). Gen. Tech. Rep. NC-234. USDA Forest Service, North Central Experiment Station, St. Paul, MN, USA, pp. 551-555.

Pitt, D., D. Weingartner et S. Greifenhagen. 2001. Precommercial thinning of trembling aspen in northern Ontario: Part 2 - Interactions with Hypoxylon canker. For. Chron. 77: 902-910.

Rink, G. 1990. Juglans cinerea L. Butternut. In : Silvics of North America. Vol. 2 Hardwoods, Burns, R.M. and Honkala, B.H. (tech. coord.), USDA For. Serv. Agr. Hdbk, pp. 386-390.

Rioux, D., M. Blais, N. Nadeau-Thibodeau, M. Lagacé, P. DesRochers, K. Klimaszewska et L. Bernier. 2018. First extensive microscopic study of butternut defense mechanisms following inoculation with the canker pathogen Ophiognomonia clavigignenti-juglandacearum reveals compartmentalization of tissue damage. Phytopathology 108: 1237-1252.

Rioux, D., P. Tanguay, K. Sambaraju, N. Nadeau-Thibodeau et P. DesRochers. 2019. L'histoire d'un chancre menaçant d'exterminer le noyer cendré. Nat. Can. 143 : 39-48. https://doi.org/10.7202/ 1060054ar

Sambaraju, K.R., P. DesRochers et D. Rioux. 2018. Factors influencing the regional dynamics of butternut canker. Plant Dis. 102: 743-752.

SAS Institute. 2008. SAS/STAT 9.2 User's Guide - The GLM Procedure (Book Excerpt). Cary, NC SAS Institute Inc., 208 pp.

SAS Institute. 2011. SAS/STAT 9.3 User's Guide. Cary, NC, SAS Institute Inc. pp. 4033-4267.

Sinclair, W. et H. Lyon. 2005. Sirococcus canker of butternut. In : Diseases of trees and shrubs. Second edition, Cornell University Press, N.Y., 116 pp.

Stringer, J.W. et R.F. Wittwer. 1985. Release and fertilization of black walnut in natural stands. In : Dawson, O.J. and A.K. Majerus (Eds.). Proceedings of the 5th Central hardwood forest conference. University of Illinois, Urbana-Champaign, IL, USA, pp. 62-67.

Stokes, M.E., C.H. Davis et G.G. Koch. 1995. Categorical data analysis using the SAS system. Cary, NC, SAS Institute Inc. pp. 163-246. Tanguay, P., M. Blais, A. Potvin, D. Stewart, D. Walker, N. Nadeau-Thibodeau, P. DesRochers et D. Rioux. 2018. qPCR quantification of Ophiognomonia clavigignenti-juglandacearum from infected butternut trees under different release treatments. For. Pathol. 48, 13 pp. https://doi.org/10.1111/efp.12418

Thibault, M. 1993. Guide du soin des arbres. Saint-Laurent, Montréal, Éditions du Trécarré, pp. 107-119.

Weber, R.W.S. 2014. Biology and control of the apple canker fungus Neonectria ditissima (syn. N. galligena) from a Northwestern European perspective. Erwerbs-Obstbau 56: 95-107. https:// doi.org/10.1007/s10341-014-0210-x

Woeste, K.E., L. Farlee, M.E. Ostry, J.R. McKenna et S. Weeks. 2009. A Forest Manager's Guide to Butternut. North. J. Appl. For. 26: 9-14. 